\title{
Experimental investigation of wake evolution behind a couple of flat discs in a hydrochannel
}

\author{
Naumov, I.V.; Litvinov, I.V. ; Mikkelsen, Robert Flemming; Okulov, Valery
}

Published in:

Thermophysics and Aeromechanics

Link to article, DOI:

$10.1134 / \mathrm{S} 0869864316050048$

Publication date:

2016

Document Version

Publisher's PDF, also known as Version of record

Link back to DTU Orbit

Citation (APA):

Naumov, I. V., Litvinov, I. V., Mikkelsen, R. F., \& Okulov, V. (2016). Experimental investigation of wake evolution behind a couple of flat discs in a hydrochannel. Thermophysics and Aeromechanics, 23(5), 657-666.

https://doi.org/10.1134/S0869864316050048

\section{General rights}

Copyright and moral rights for the publications made accessible in the public portal are retained by the authors and/or other copyright owners and it is a condition of accessing publications that users recognise and abide by the legal requirements associated with these rights.

- Users may download and print one copy of any publication from the public portal for the purpose of private study or research.

- You may not further distribute the material or use it for any profit-making activity or commercial gain

- You may freely distribute the URL identifying the publication in the public portal 
Thermophysics and Aeromechanics, 2016, Vol. 23, No. 5

DOI: $10.1134 / \mathrm{S} 0869864316050048$

\title{
Experimental investigation of wake evolution behind a couple of flat discs in a hydrochannel"
}

\author{
I.V. Naumov ${ }^{1}$, I.V. Litvinov ${ }^{1}$, R.F. Mikkelsen ${ }^{2}$, and V.L. Okulov ${ }^{1,2}$ \\ ${ }^{1}$ Kutateladze Institute of Thermophysics SB RAS, Novosibirsk, Russia \\ ${ }^{2}$ Technical University of Denmark, Lyngby, Denmark \\ (Received October 30, 2015; in revised form December 24, 2015) \\ E-mail: naumov@itp.nsc.ru
}

\begin{abstract}
The decay of a far wake and its turbulent fluctuations behind two thin discs of the same diameter $D$, oriented normal to the incident flow, have been studied using the Particle Image Velocimetry (PIV). The experimental study was carried out in a water flume $\left(\operatorname{Re} \approx 2 \cdot 10^{5}\right)$ with varying distances between the discs $\left(L_{x}=4-8 D\right)$ and their axes shift relative to each other $(0,0.5 D$ and $1 D)$. It is found that the velocity deficit behind two discs depends weakly on $L_{x}$, and at $L_{x}>40 D$, it becomes indistinguishable from the level of turbulent fluctuations of the incident flow. It is found that the decay of the average velocity deficit and its turbulent fluctuations in a wake of a tandem of discs can be described by the same analytical dependence with exponent $-2 / 3$ as for the wake decay of a single disc. However, at the same distance downstream, the value of deficit behind two discs is substantially higher than the corresponding value behind a single disc. Velocity fluctuations in a far wake behind a pair of discs depend weakly on longitudinal dimension $L_{x}$, but at the same time, in contrast to the velocity deficit, their level does not differ significantly from the level of fluctuations behind a single disc.
\end{abstract}

Key words: wake behind a bluff body, level of turbulence of the incident flow, pair of discs, velocity deficit, Particle Image Velocimetry (PIV).

\section{Introduction}

Interaction between the wakes of two or more fixed bluff bodies is typical of various technical areas. It draws particular attention when assessing the wind loads on the repeating elements in the construction of bridges, buildings, chimney pipes, and other civil facilities. Each specific technical application is characterized by certain geometry, and, as a consequence, the specific behavior of the wake, but their identification requires first the examination of flow behavior around some canonical configurations consisting, for example, of two or more round cylinders and spheres [1], rectangular [2] and circular [3] plates. The similar sets of repeating samples can be useful for solving various engineering problems. In this regard, the study of considered new configurations of a pair of identical discs is an important and interesting problem for the mentioned applications.

\footnotetext{
${ }^{*}$ Research is financially supported by the Russian Foundation for Basic research grant (Project No. 14-29-00093).
}

(C) I.V. Naumov, I.V. Litvinov, R.F. Mikkelsen, and V.L. Okulov, 2016 
A new impetus to the study of interaction between the wakes behind a system of identical bodies was given by intensive development of wind power stations, where the same-type wind turbines were arranged one behind another with some shift downstream. In this light, the problem of modeling interactions and the development of wakes behind a pair of flat discs gets a new relevance. Indeed, to model the wind turbine systems, due to their complexity the impellers are often approximately replaced by various systems of loaded discs, especially for numerical simulation of interaction between wind turbines in the large parks $[4,5]$. Obviously, it is necessary to ground the validity of this approach for modeling. This requires the comparison of wake development behind different configurations of discs and turbine rotors. Such experimental comparison has been undertaken only for the single samples. It is shown in [6] that the wakes behind a rotating rotor model and porous discgrid become indistinguishable downstream at the distances more than three of their diameters. In the context of data of comparisons, the systematic experimental studies on development of a far wake behind the systems of several bodies have not been carried out yet. The experiments on the effect of mutual arrangement of a pair of thin discs on the development of far wake performance, described in this paper, will help, in particular, to the further development and verification of various numerical models of interaction of wakes behind the wind turbine systems at wind stations.

In literature, there are many data on the flow around a single disc with diameter $D$ at different Reynolds numbers, defined by the freestream velocity and diameter of the tested model. In the standard wind tunnels at high Reynolds numbers $\left(\geq 10^{4}\right)$, the wake, as a rule, can be examined only on the short distances of up to ten diameters $D$ of the model even at the maximal freestream velocities. This is due to the necessity of using the large models. When studying the wakes in these tunnels at the distance of several tens and, even more, hundreds of calibers, the size of the tested body should be reduced by the same times; therefore, the Reynolds numbers of the initial flow will decrease proportionally. In particular, these restrictions explain the fact that in a wide range of Reynolds numbers in many experimental studies of a wake behind the bluff bodies, the main attention is paid to the near wake of up to 10 diameters (e.g., see [7]). In calculations of [8], the fields of research were the same as in [7]. The characteristics of a far wake are rarely described in literature (e.g., see [9]), although it is known that even in the extreme far wake behind a disc $(x / D>400)$, the large-scale vortex structures are kept and continue developing without the small-scale vortices. Often it is difficult to estimate because intensity of freestream turbulence is of the same order as the fluc-tuations in the wake velocity with their slow decay downstream [9]. The experiments carried out in a special wind tunnel with a very low level of fluctuations have shown that the far wake behind the disc $(10<x / D<$ $<150)$ has the properties of self-similarity $[10,11]$. To confirm the similarity properties of the wake in the different environment and at different Reynolds numbers, the experiments with a single disc were repeated in a water flume [12]. In both studies behind a single disc, the test data showed excellent consistency with the analytical power dependences of far wake selfsimilarity, describing both the turbulent wake decay and expansion of its profile.

The authors do not know the works which dealt directly with the experimental studies of a far wake behind the similar flat discs. The literature presents only the studies on the near wake [1-3]. The focus of these studies is concentrated on determining the effect of a distance between bodies $L$ on the scenario of the development of large-scale fluctuations of the near wake and changes in their Strouhal numbers in comparison with the flow around the single bodies $[2,3]$. The distinctive feature here is weak dependence between the frequency of lowfrequency fluctuations and an increase in the distance between the bodies after some critical value. That is, for relatively short distances between two plates, which do not exceed this value, there are different regimes $[13,14]$ with different values of the dominant frequencies, but with an increase in the distance $(x / D>8)$, the flow characteristics change slightly. They become close to the flow characteristics behind a single disc. The Strouhal numbers, for example, are almost the same $[2,14]$ and depend weakly on the Reynolds number in the range from $10^{4}$ to $5 \cdot 10^{5}$. 
The aim of this work is the experimental study of a far wake behind two discs at their coaxial and out-of-line arrangement. The out-of-line arrangement is often used in many practical applications, for example, when the direction of a freestream (wind) is changed. The study intended to find how a couple of discs and their shifted out-of-line arrangement affect the law of far wake decay and its turbulent fluctuations. It was planned to compare the data with the results of the previous experiment for a single disc [12].

\section{Experimental methods and conditions}

Experiments on the flow velocity decay behind a couple of discs were carried out in a water flume at the Technical University of Denmark. The flume length was $35 \mathrm{~m}$, width was $3 \mathrm{~m}$, and working height was $1 \mathrm{~m}$. The most complete description of the experimental conditions in this water flume can be found in $[15,16]$, when the optical measurement of the flow velocity behind a model of the wind turbine rotor were carried out using laser Doppler anemometry (LDA) and Particle Image Velocimetry (PIV). The constant value of the freestream velocity $\left(U_{0}=0.54 \mathrm{~m} / \mathrm{s}\right)$ was kept by maintaining the constant flow rate of $1.4 \mathrm{~m}^{3} / \mathrm{s}$ through a closed circuit of the flume. Fluctuations in flow rate during the experiment did not exceed $2 \%$. The thickness of a boundary layer $(\approx 0.2 \mathrm{~m})$ and the level of turbulent fluctuations $\left(U_{\mathrm{rms}} / U_{0} \approx 2.5 \%\right.$ ) of the undisturbed flow in the central part of the flume have been determined previously, and they are described in detail in [15-17]. In this paper, to investigate the far wake behind the tandem, two identical discs made of organic glass with diameter $D=300 \mathrm{~mm}$ and thickness $h=10 \mathrm{~mm}$ were used (Figs. 1 and 2).

The discs were fixed by the holders used in [15] and mounted on the platforms moved over the flume. The disc axes were in the middle of the channel cross section at the height of $0.5 \mathrm{~m}$ from the bottom and at $1.5 \mathrm{~m}$ from the flume wall to minimize the influence of irregularity profile of the freestream velocity. The distance between discs $L_{x}$, which takes the values of $4 D, 6 D$, and $8 D$ was varied during the study. To perform optical measurements, the measuring section of 3-m length, whose walls and bottom were made of glass, was located at the distance of $20 \mathrm{~m}$ from the beginning of the flume. To measure the velocity, we used the SPIV Dantec, whose application features are described, for example, in $[16,17]$. To form a light sheet, the pulsed laser Litron 200-15 PIV Nd:Yag with $200 \mathrm{~mJ}$ energy per a pulse and shooting frequency of up to $15 \mathrm{~Hz}$, was used as a lighter. The laser sheet of 2-mm thickness passed vertically in the direction of movement of the main incident flow through the flume bottom and disc axes (Fig. 1). Two eight-bit CMOS-cameras SpeedSense 1040 with resolution

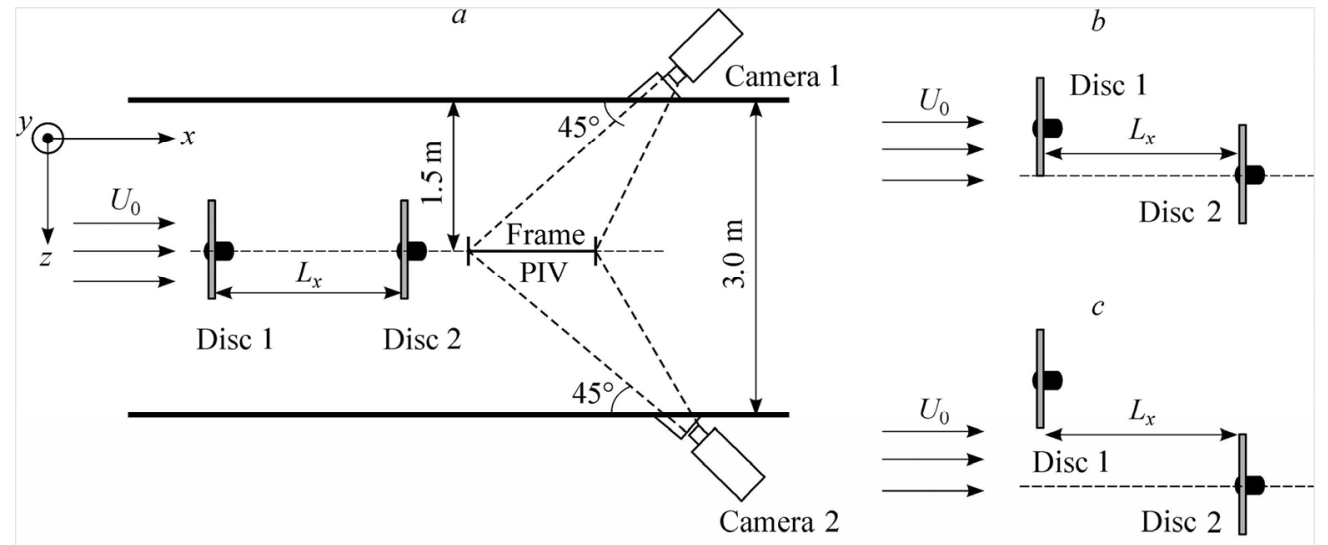

Fig. 1. Scheme of experiment on measurement of velocity fields behind the second disc by PIV for different spatial locations of two discs.

$a-$ co-axial location of discs, $b-$ shift by a half of diameter $\left(L_{y}=0.5 D\right)$, $c$ - shift by one diameter $\left(L_{y}=1 D\right)$. 


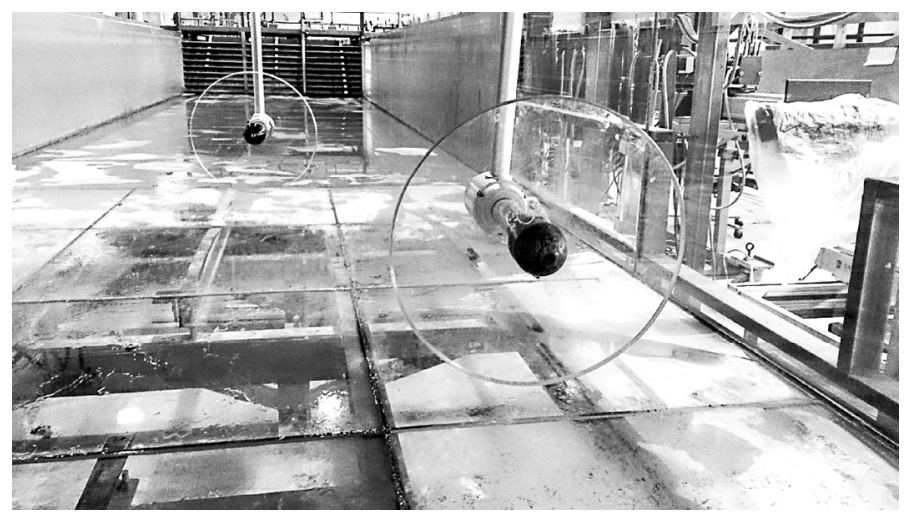

Fig. 2. Picture of spatial location of discs in a flume.

of $2320 \times 1726$ and maximal frequency of full frame capture of up to 193 frames per second were used in experiments. The size of the measuring PIV window was $732 \times 430 \mathrm{~mm}$. To calculate the three-dimensional velocity field, Dantec Dynamic Studio Version 2.21 software was used.

During the measurements, the cameras were mounted at different sides of the flume at the angle of $45^{\circ}$ (Fig. 1). To reduce image distortion, the optical prisms, filled with water, were mounted between the camera and channel wall; this provides parallel arrangement of the plane camera matrix and air-glass-water interface. Since the cameras were not positioned frontally, but at the angle to the light section, to focus the entire light region on the matrix plane, the unit of independent adjustment of receiving objective and camera matrix, recording the image, were used. Calibration of stereo-measuring equipment described in detail in [16, 17] allowed avoiding optical aberrations as compared to a planar measuring system [12] and provided the opportunity to calculate the averaged three-dimensional velocity field and turbulent fluctuations with an error of $3-5 \%$. In doing so the error in determining the longitudinal velocity component was guaranteed below $2 \%$.

Each measuring PIV-window was positioned due to the displacement of a movable platform with the fixed discs (along the $x$-axis as shown in Fig. 1) upstream by $550 \mathrm{~mm}$ along the flume. Disc displacement along the channel allowed a change in the measuring windows without changing the position of the optical system (laser sheet and recording image camera). Axis $y$ passed through the line connecting the discs centers, and the front edge of the second disc was the origin along $x$-axis.

The resulting velocity fields were obtained for each measuring window by averaging 200 instantaneous velocity fields measured with the frequency of $10 \mathrm{~Hz}$. Due to processing the images by the Dynamic Studio program in the plane of $x-y$ light sheet, the pattern of the flow around two discs was defined by sewing and uniform cutting of the zones of measuring window overlapping to distance $x / D=45$. The decay of averaged deficit of longitudinal velocity component and fluctuating characteristics of the flow behind the second disc downstream were calculated by velocity distributions for three different $L_{x}$ with coaxial disc location and offset of the first disc axis by $0.5 \mathrm{D}$ and $1.0 \mathrm{D}$ across the flume.

\section{Experimental results and discussion}

When analyzing the experimental data, it is necessary to determine velocity distribution in a wake or, at least, it is necessary to know its maximal deceleration - deficit:

$$
\frac{\Delta U(x)}{U_{0}}=\frac{\max \left(U_{0}-U_{\text {axial }}(x)\right)}{U_{0}},
$$

where $U_{0}$ is velocity of the freestream, and $U_{\text {axial }}$ is the longitudinal velocity component of a wake behind the disc. 


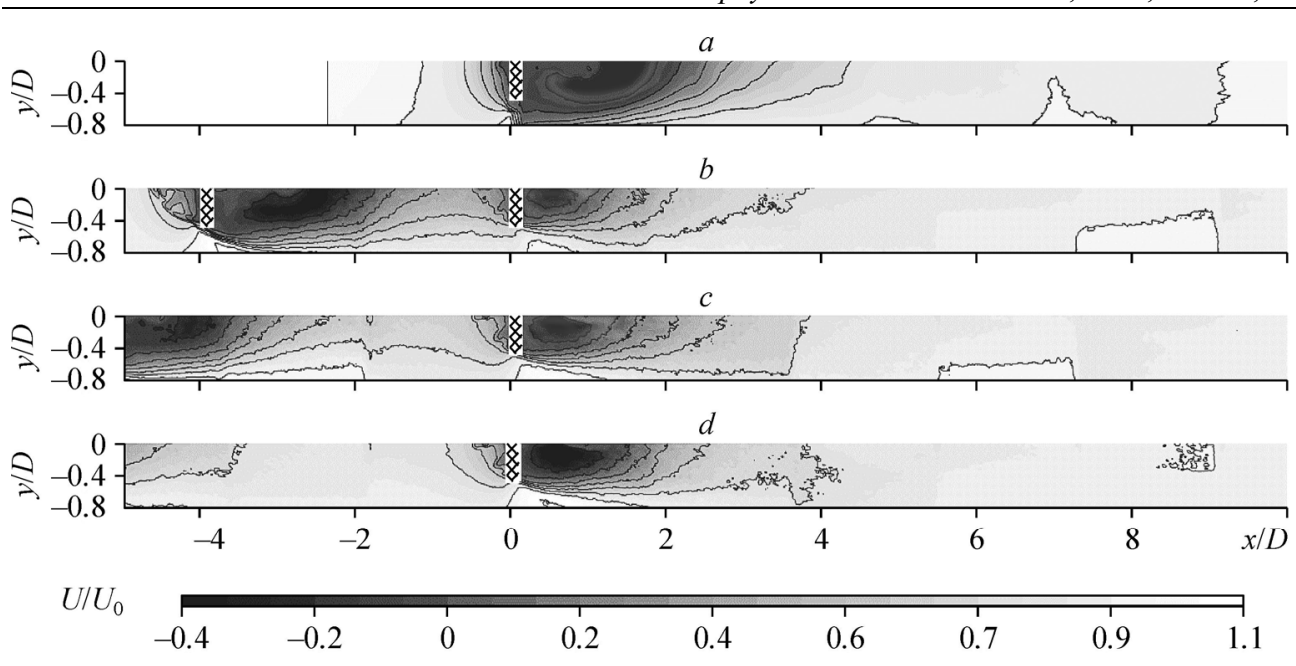

Fig. 3. Comparison of normalized longitudinal component of average velocity behind a single disc (a) and two coaxial discs at $L_{x}=4 D(b), 6 D(c)$ and $8 D(d)$.

Decays of the average velocity field in a vertical cross section behind a single disc below its axis [12] and in the same cross section behind a coaxial pair of discs with different longitudinal sizes $L_{x}=4 D, 6 D$, and $8 D$ are compared in Fig. 3 .

A far wake behind the disc is the flow, rather complicated for diagnostics due to low values of maximal average velocity deficit (1) downstream in comparison with disturbances of the freestream. Therefore, the PIV data on distribution of longitudinal velocity components are presented here only in the near wake up to the distances downstream $x / D<10$ in the region, where the plotted isolines allow contrast visualization of the flow around the discs. Based on these data, it is possible to make a statement about a weak impact of perturbation caused by the fastening devices on the near wake and its complete disappearance, when approaching $x / D=10$ and, respectively, at the points of the far wake for $x / D>10$. As can be seen from Fig. $3 b$, for a pair of discs at $L_{x}=4 D$, the area of reverse flow behind the second disc in the wake of the first one reduced its axial dimensions from $2 D$ to $D$ as compared to a single disc (Fig. $3 a$ ). With an increase in the longitudinal dimension of the pair to $L_{x}=8 D$ (Fig. $3 d$ ), the zone of reverse flow increases; thus, influence intensity of the first disc wake decreases, when it removes from the second disc. Using these results, we will analyze the characteristics of the flow incoming to the second disc. In particular, we will determine the profile of longitudinal velocity component and level of its fluctuations at the distance of one diameter $D$ in front of the disc for the flow incoming to the second disc (Fig. 4). We will compare the profiles of velocity and its fluctuations for a single disc, which also slightly change in front of the last; they are indicated in the figure by the dark symbols. In comparison with the data for a single disc, it can be seen that in the case of $L_{x}=8 D$, the influence of a wake of the first disc is minimal. The profile of longitudinal velocity component is reduced to the level of $80 \%$ of the flow velocity incoming to a single disc. For a less longitudinal distance between the discs $\left(L_{x}=4 D\right)$, the velocity deficit on the axis reaches $40 \%$, which, of course, has less influence on formation of a stagnation zone of the reverse flow. Comparison of the profiles of standard deviations from the mean velocity before the second disc (Fig. 4b) also demonstrates an increase in the level of fluctuations before the second disc at their approaching. For example, for a minimal longitudinal size of a pair $\left(L_{x}=4 D\right)$, the standard deviations reach $20 \%$ of the velocity of free stream.

Let us now consider the nature of fluctuations after the stagnation zone in the wake behind the pair of discs. In this experimental study, it was found that at longitudinal dimensions of the pair $L_{x}>4 D$ at some distance from its second disc within $5<x / D<15$, the pronounced 

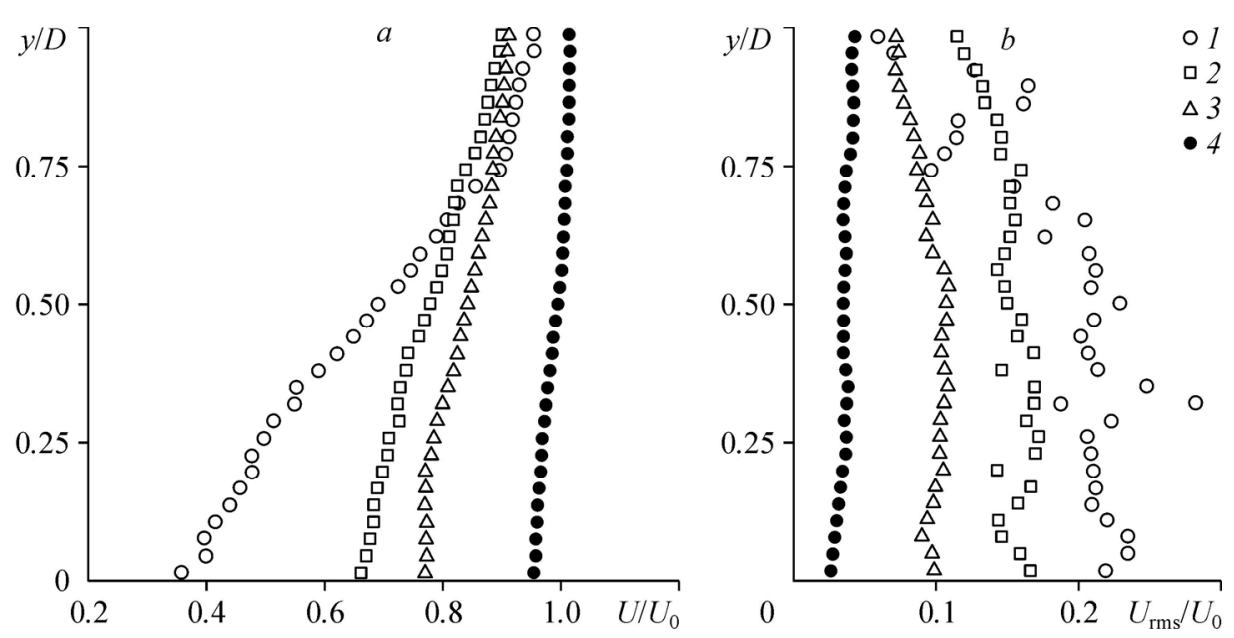

Fig. 4. Profile of longitudinal velocity component at distance $D$ before the second disc.

$a$ - averaged velocity, $b-$ mean-square deviation of velocity; two discs at $L_{x}=4 D(1), 6 D(2), 8 D(3)$, single disc (4).

low-frequency fluctuations can be distinguished in the wake. Like behind a single disc, pronounced dominant frequency $f=0.3 \mathrm{~Hz}$, corresponding to Strouhal number $\mathrm{St} \sim 0.14$, calculated by the disc diameter and free flow velocity, is distinguished in fluctuation spectrum behind the pair of discs. This is consistent with the results of $[7,8]$.

According to [9], to study the far wake, we approximate dependence of velocity deficit on distance $x / D$ by formula of the $G$-model determined in $[10,11]$ :

$$
G(x)=\Delta U(x) / U_{0}=a\left(x-x_{0}\right)^{-2 / 3},
$$

where $a$ and $x_{0}$ are determined by the type of the bluff body, and velocity deficit $\Delta U$ on the axis is determined by equation (1). Distribution of velocity deficit normalized to the incident flow velocity along the disc axis away downstream for three longitudinal dimensions of the disc pair $L_{x}$ is shown in Fig. 5. Hereinafter, in Figs. 6-8, the symbols indicate experimental data obtained by the PIV-averaged velocity fields, and lines represent approximating curves of the G-model obtained by formula (2). In all cases, the velocity deficit reaches its maximal value at point $x / D=4$, and then, after the zone of reverse flow behind the second disc, it decreases rapidly with the following movement downstream and takes the constant value determined by the degree of incident flow fluctuations like the measured velocity deficit behind a single disc [12]. Experimental points of longitudinal velocity deficit along the axis of a disc pairs downstream fit well to the proposed power dependence $\left(\sim x^{-2 / 3}\right)$ for turbulent wake decay behind the blunt bodies; only the empirical coefficients in (2) differ. For the single disc in [12], these factors have been identified as $a=0.31$ and $x_{0}=3.2$, then for a pair of discs with $L_{x}=4 D, 6 D$ and $8 D$, these coefficients take other values, very close to each other, which can be approximated by the average curve at $a=0.49$ and $x_{0}=2.1$. According to the diagram, within $5<x / D<40$, the integral curve characterizing velocity deficit behind the disc tandem is located higher than the curve for the velocity deficit behind a single disc. We can also note the existence of a weak dependence of the far wake on the distance between the discs; with an increase in this distance, the wake shape approaches the shape of the wake behind a single disc. Like behind a single disc, velocity deficit behind a pair of discs for all longitudinal dimensions $L$ at distance $x / D \approx 40$ takes constant value $\Delta U(x) / U_{0} \approx 0.05$, determined by 


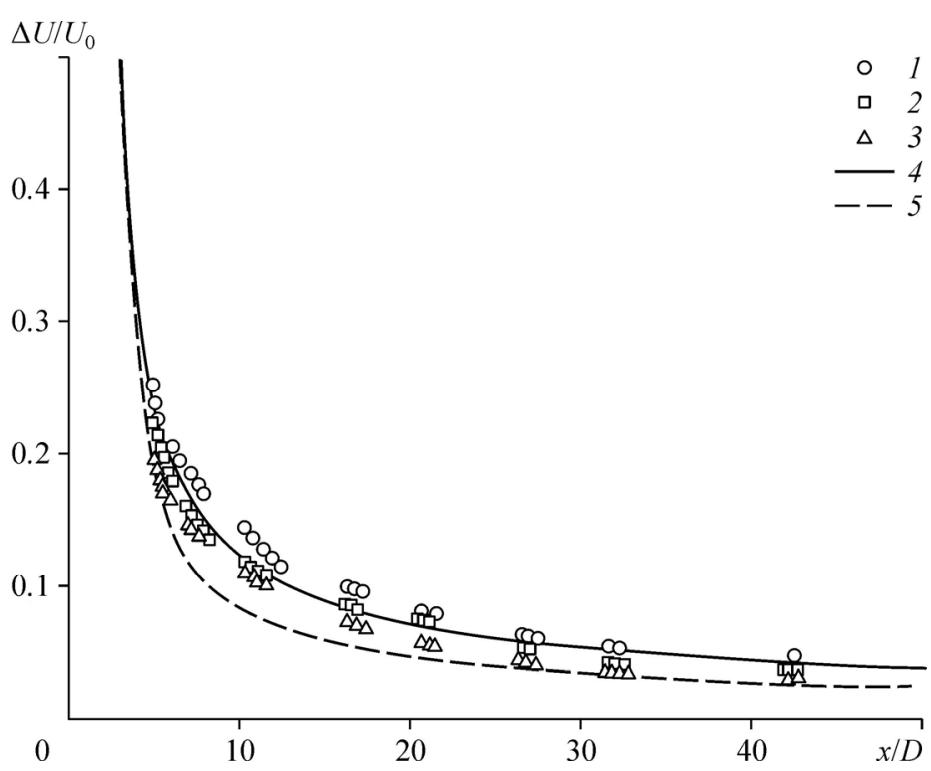

Fig. 5. Decay of deficit of the longitudinal velocity component normalized by the incident flow velocity behind a pair of co-axial discs at $L_{x}=4 D(1), 6 D(2)$, and $8 D(3), L_{y}=0$ (4) ( $G$-model) and single disc (5) from [12].

the level of the freestream fluctuations. Note that for $L_{x}=8 D$, the velocity deficit starts coinciding with the deficit for a single disc already at distances $x / D>30$.

We investigate the effect of a cross shift between the axes of the pair of discs on far wake decay. Figures 6 and 7 show experimental dependences of a change in the deficit of longitudinal velocity component behind the discs at $L_{x}=4 D, 6 D, 8 D$ and $L_{y}=0.5 D, 1 D$; comparisons with approximation curves (2), according to power dependence $-2 / 3$, are presented. The coefficients in model (2) for deficit decay behind the second take values $a=0.42$ and $x_{0}=2.67$ for $L_{y}=0.5 D$ and $a=0.34$ and $x_{0}=2.9$ for $L_{y}=1 D$.

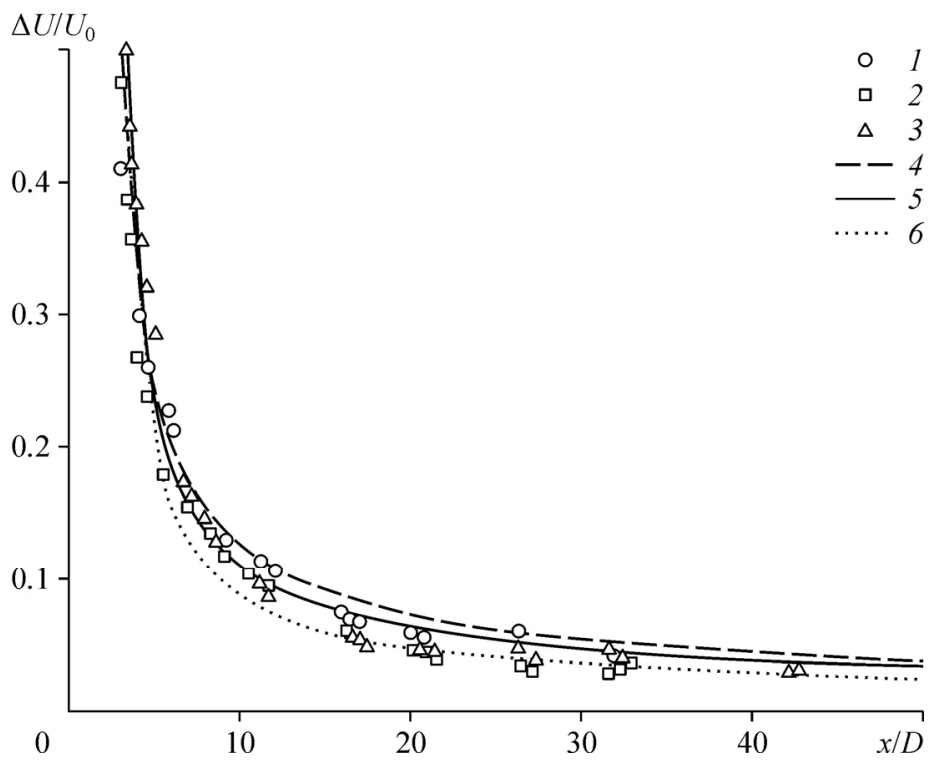

Fig. 6. Decay of normalized velocity deficit behind the discs at $L_{x}=4 D(1), 6 D(2), 8 D(3)$ at axes shift by $L_{y}=0.5 D$ and comparison with the approximating curves.

Two discs at $L_{y}=0(4), 0.5$ (5), single disc (6). 


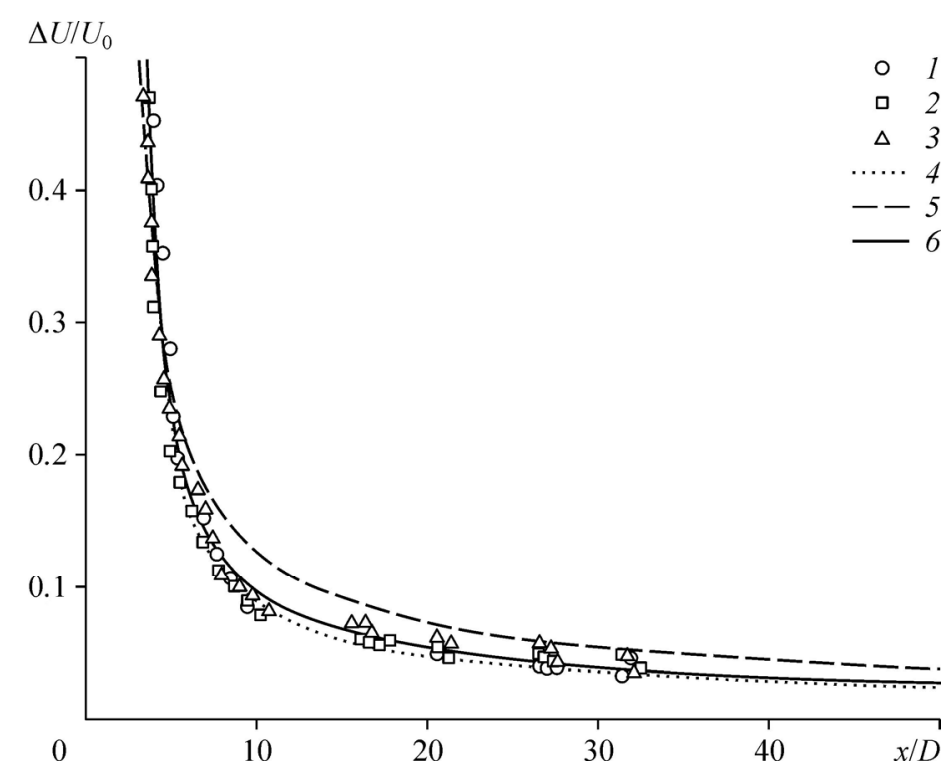

Fig. 7. Decay of normalized velocity deficit behind the discs at $L_{x}=4 D(1), 6 D(2), 8 D(3)$ at axes shear by $L_{y}=1 D$ and comparison with the approximating curves. Single disc (4), two discs at $L_{v}=0(5), 1 D(6)$.

While analyzing distribution of the velocity deficit downstream under the considered disc location, we can conclude that there is a displacement of the deficit curve towards a single disc at an increase in the shift between the disc axes. The curve for shift $L_{y}=0.5 D$ is closer to the curve for the coaxial discs, but with an increase in the shift to $L_{y}=1 D$, the deficit curve almost completely coincides with the decay of the longitudinal velocity component for a single disc. This is an expected result because in the last case, the second disc is entirely in the zone of the freestream. We should also note weak dependence of velocity deficit decay on longitudinal size of the disc pair $L_{x}=4 D, 6 D$, and $8 D$.

Finally, we will consider the question about the decay of velocity fluctuations behind two co-axial discs. The symbols in Fig. 8 show distribution of fluctuations of longitudinal velocity component behind the second disc downstream at different longitudinal distances between

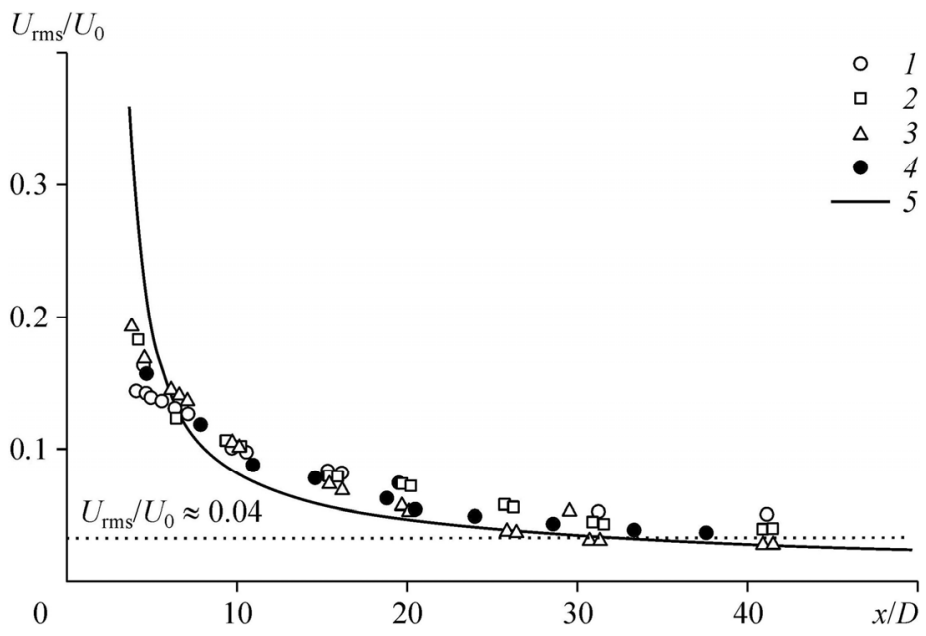

Fig. 8. Decay of normalized RMS deviation of velocity behind disc tandem at $L_{x}=4 D(1), 6 D$ (2), and $8 D(3)$, behind a single disc (4) borrowed from [12], and behind a single disc according to the $G$-model (5). 
a pair of discs. The highest level of fluctuations is predictably located in the region located just behind the area of the reverse flow of the second disc, where the maximal velocity gradient is observed. For comparison, the solid line in Fig. 8 shows approximation of fluctuation decay by means of model (2) used to describe velocity fluctuations in a wake of a single disc [12].

The level of velocity fluctuations behind a pair of single-axis discs for $L_{x}=4 D, 6 D$, and $8 D$ after $x / D>8$ is almost identical and slightly exceeds the level of approximating curve for the decay of fluctuations in a wake of single disc [12]. Thus, the standard deviation of velocity fluctuations behind the pair of coaxial discs, as in the case of a single disc, has a similar character with attenuation of velocity deficit (2) by power dependence $-2 / 3$, i.e., $U_{\mathrm{rms}} / \Delta U \approx 1$. This conclusion is also valid even at transverse displacement of the disc axes in the pair. It is stated in $[10,11]$ that the measurement of velocity deficit becomes critical for diagnostics, when it reduces to the level of turbulent fluctuations of the freestream, that is, the wake and its pulsations become indistinguishable against the background of external disturbances, and experimental data reach their constant values. This was proved in [12] and in the current experiment: these restrictions in determining decay of deficit and fluctuations of longitudinal velocity component have been determined expectedly. At distance $x / D \approx 40$, velocity deficit and fluctuation level reached their constant values $\left(\Delta U / U_{0} \approx U_{\text {rms }} / U_{0} \approx 0.04\right)$, which corresponded to the level of fluctuations of the freestream for the experimental conditions of the water channel.

Another interesting fact is that the distance between the discs has a small effect on the level of velocity deficit in a far wake, but the behavior of the near wake behind a second disc in the pair is significantly dependent on this distance. It is seen from the analysis of velocity profiles and its fluctuations before the second disc (Fig. 4) that they are very uneven and different. It turned out that this different non-uniformity before the second disc in the pair does not affect the laws of far wake decay and its fluctuations, but its influence on the near wake and size of stagnation zone behind the second disc is very strong. There arises a conclusion that location of the first disc has a strong influence on the development of a far wake, and location of the second disc influences the development of a near wake.

\section{Conclusion}

In this paper, the decay of a far wake and level of its turbulent fluctuations behind a pair of thin discs with diameter $D$, normal to the freestream, were studied using particle image velocimetry. Experimental study was performed in a water flume $\left(\operatorname{Re} \approx 2 \cdot 10^{5}\right)$ with varying longitudinal distance between the discs $\left(L_{x}=4 D, 6 D\right.$, and $\left.8 D\right)$ and different transverse shift between the disc axes $(0,0.5 D$, and $1 D)$.

When studying the far wake behind a pair of discs for all spatial configurations, it was determined that the law of decay of longitudinal component of the averaged velocity is subject to the well-known power dependence $\sim x^{-2 / 3}$, determined previously for streamlining the individual bluff bodies at relatively high Reynolds numbers. The value of the maximal velocity deficit in a wake behind the pair of two discs was larger than in the wake behind a single disc. However, the difference between intensity of a far wake for different distances between the discs $\left(L_{x}=4 D, 6 D\right.$, and $8 D$ ) was insignificant as compared with the difference between the wakes behind a pair of discs and a single disc. In contrast to this conclusion, the effect of a distance between the discs was essential for the development of the near wake.

\section{References}

1. M.M. Zdravkovich, Review of flow interference between two circular cylinders in various arrangements, Trans. ASME. J. Fluids Engng, 1977, Vol. 99, No. 4, P. 618-633.

2. F. Auteri, M. Belan, G. Gibertini, and D. Grassi, Normal flat plates in tandem: An experimental investigation, J. Wind Engng Ind. Aerodyn., 2008, Vol. 6, No. 96, P. 872-879.

3. H. Hacsşevki and A. Teimourian, Comparison of flow structures in the wake region of two similar normal flat plates in tandem and a square cylinder, Exp. Therm. Fluid Sci., 2015, Vol. 69, P. 169-177. 
4. F. Porté-Agel, Y.T. Wu, and C.H. Chen, Numerical study of the effects of wind direction on turbine wakes and power losses in a large wind farm, Energies, 2013, Vol. 6, No. 10, P. 5297-5313.

5. X. Yang, S. Kang, and F. Sotiropoulos, Computational study and modeling of turbine spacing effects in infinite aligned wind farms, Physics of Fluids, 2012, Vol. 24, No. 11, P. 115107-1-115107-28.

6. S. Aubrun, S. Loyera, P.E. Hancock, and P. Hayden, Wind turbine wake properties: Comparison between a nonrotating simplified wind turbine model and a rotating model, J. Wind Engng Ind. Aerodyn., 2013, Vol. 120, P. 1-8.

7. A. Roshko, On the wake and drag of bluff bodies, J. Aeronautical Sci., 1955, Vol. 2. P. 124-132.

8. J. Yang, M. Liu, G. Wu, W. Zhong, and X. Zhang, Numerical study on coherent structure behind a circular disk, J. Fluids Struct., 2014, Vol. 51, P. 172-188.

9. B.G. Novikov, Effect of small total pulse on development of a wake behind the self-propelled bodies, Thermophysics and Aeromechanics, 2009, Vol. 16, No. 4, P. 561-583.

10. P.B. Johansson, W.K. George, and M.J. Gourlay, Equilibrium similarity, effects of initial conditions and local Reynolds number on the axisymmetric wake, Phys. Fluids, 2003, Vol. 15, No. 3, P. 603-617.

11. P.B. Johansson and W.K. George, The far downstream evolution of the high-Reynolds number axisymmetric wake behind a disk. Part 1. Single-point statistics, J. Fluid Mech., 2006, Vol. 555, P. 363-385.

12. I.V. Naumov, I.V. Litvinov, R.F. Mikkelsen, and V.L. Okulov, Investigation of a wake decay behind a circular disc in a hydro channel at high Reynolds numbers, Thermophysics and Aeromechanics, 2015, Vol. 22, No. 6 , P. 657-665.

13. Y.N. Nakamura, Vortex shedding from bluff bodies and a universal Strouhal number, J. Fluids Struct., 1996, Vol. 10, P. 159-171.

14. J.P. Bentley and A.R. Nichols, The mapping of vortex fields around single and dual bluff bodies, Flow Meas. Instrum., 1990, Vol. 1, P. 278-286.

15. V.L. Okulov, I.V. Naumov, R.F. Mikkelsen, I.K. Kabardin, and J.N. Sørensen, A regular Strouhal number for large-scale instability in the far wake of a rotor, J. Fluid Mech., 2014, Vol. 747, P. 369-380.

16. I.V. Naumov, V.V. Rahmanov, V.L. Okulov, K.M. Velte, K.E. Mayer, and R.F. Mikkelsen, Flow diagnostics downstream of a tribladed rotor model, Thermophysics and Aeromechanics, 2012, Vol. 19, No. 2, P. 171-181.

17. I.V. Naumov, R.F. Mikkelsen, V.L. Okulov, and J.N. Sørensen, PIV and LDA measurements of the wake behind a wind turbine model, J. Phys.: Conference Series, 2014, Vol. 524, No. 1, P. 12168-12177. 